\title{
Adaptive Generalized Vector Median Filter
}

\author{
Bogdan Smolka \\ Silesian University of Technology, Department of Automatic Control, \\ Akademicka 16, 44-100 Gliwice, Poland \\ smolka@ieee.org
}

\begin{abstract}
In this paper, a new adaptive filter intended for the attenuation of impulse noise in color images is proposed. The new filtering design is based on the concept of a peer group of pixels sharing similar chromatic properties. The novel approach adaptively determines the size of the peer group which minimizes the aggregated distance to the peer group members, utilizing the Fisher linear discriminant. The analysis of the obtained noise reduction results leads to the conclusion that the new filter is capable of reducing even strong impulse noise, while preserving and even enhancing the edges of color images. This unique property of the proposed filtering design is shown on examples of color biomedical images.
\end{abstract}

Keywords: Color image processing, impulse noise reduction, image enhancement.

\section{Introduction}

The Vector Median Filter (VMF) is the most popular vectorial operator used for the removal of impulses injected into the color image by the noise process 11. This filter is quite efficient at reducing the impulses, retains sharp edges and linear trends, however it does not preserve fine image structures, which are treated as noise and therefore generally the VMF tends to produce blurry images. This unwanted feature of the VMF is very important as much of the image information is contained in its edges, and sharp edges are pleasing to humans and are desirable for machine processing.

Many edge enhancement techniques that counteract blur without the knowledge about its model are based on linear filters which emphasize the highfrequency image components performing the high-pass filtering in the spatial or frequency domain. The drawback of these simple techniques is that they strongly amplify the image noise and texture and produce annoying overshoots and ringing effects at sharp edges. Also the deconvolution algorithms fail in the presence of noise and in the cases when the knowledge about the degradation model is not available.

One of the most popular technique intended for noise reduction, while preserving edges is the anisotropic diffusion, whose aim is to encourage intraregion smoothing and to inhibit blurring of edges [2. This powerful method has been successfully applied to color images contaminated by Gaussian noise, however 
its main drawback is its difficulty to cope with impulse noise, which is being retained, as it is treated like a strong edge, which should be preserved [3].

Another solution to the problem of image quality improvement with edge enhancing abilities is provided by the shock filters, which are based on the idea to apply locally either a dilation or an erosion process, depending on whether the pixel belongs to the in influence zone of a local maximum or a minimum [4]. This filter class creates a sharp discontinuity at image edges and produces piecewise constant segmentation of the image, however it is not able to enhance the image in the presence of impulse noise, as the impulses are treated as local extrema.

The described above filtering schemes exemplify the main difficulty when enhancing images polluted by impulses. The majority of the well known techniques which are able to suppress the impulse noise tend to blur the image and those with edge enhancing properties preserve or amplify the outliers introduced by noise process. As a result much research has been devoted to the construction of filters which can cope with noise while simultaneously preserving image details and enhancing edges in the gray-scale [5] and color images [6, 7].

In this paper a solution to the problem of image noise filtering with edge enhancing abilities is proposed. Extending the VMF using the peer group concept introduced in [8, it is possible to efficiently remove impulse noise while sharpening the color image edges. The proposed filtering design is comparable in noise reduction with the $\mathrm{VMF}$, preserves much better image details and what is the most important contribution of the paper, produces images with sharp edges.

\section{Adaptive Generalized Vector Median Filter (AGVMF)}

To remove the impulse noise from color images various filtering approaches based on the order statistics theory have been proposed. The most popular filtering class operating on a sliding window is based on the vector sample ordering [6].

The reduced or aggregated ordering scheme assigns an aggregated dissimilarity measure to each color pixel from the filtering window $W$. The aggregated dissimilarity measure assigned to pixel $\boldsymbol{x}_{i}$ is defined as $R_{i}=\sum_{j=1}^{n} \rho\left(\boldsymbol{x}_{i}, \boldsymbol{x}_{j}\right)$, $\boldsymbol{x}_{i}, \boldsymbol{x}_{j} \in W$, where $\rho(\cdot)$ denotes the distance between the color pixels belonging to the filtering window $W$ containing $n$ pixels. The scalar accumulated dissimilarity measures are then sorted and the associated vectors can be correspondingly ordered. The vector median of a set of vectors from $W$ is defined as the vector $\boldsymbol{x}_{(1)}$ from $W$ for which the sum of distances to all other vectors belonging to $W$ is minimized: $\boldsymbol{x}_{(1)}=\arg \min _{\boldsymbol{x} \in W} \sum_{j=1}^{n} \rho\left(\boldsymbol{x}, \boldsymbol{x}_{j}\right)$.

The notion of the vector median can be generalized, so that the filter output will be the vector $\boldsymbol{x}_{(1)}^{\alpha}$ for which the sum of $\alpha$ smallest distances to other vectors from $W$ is minimized. Of course for $\alpha$ equal to the number of pixels $n$ in the filtering window, the standard VMF is obtained.

If the distance between the vector $\boldsymbol{x}_{i}$ and $\boldsymbol{x}_{j}$ is denoted as $\rho_{i, j}$, then we can order the set of distances $\rho_{i, j}$, for $j=1, \ldots, n$ and obtain the following sequence: $\rho_{i}^{(1)} \leq \ldots \leq \rho_{i}^{(\alpha)} \leq \ldots \leq \rho_{i}^{(n)}$, where $\rho_{i}^{(k)}$ is the $k$-th smallest distance from $\boldsymbol{x}_{i}$. For each pixel in the filtering window the cumulated sum $R_{i}^{\alpha}$ is calculated as: 


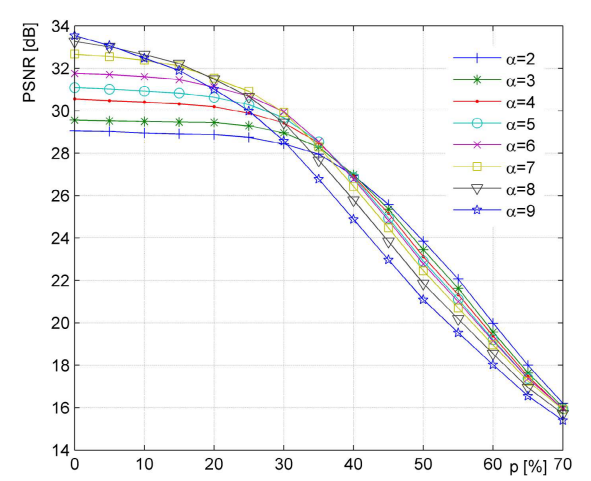

Fig. 1. Dependence of the PSNR on the value of the $\alpha$ parameter for a color test image LENA contaminated with impulsive noise of intensity $p$. The apparently weak filtering efficiency for low levels of noise is caused by the sharpening of edges which is treated by the PSNR as a distortion.

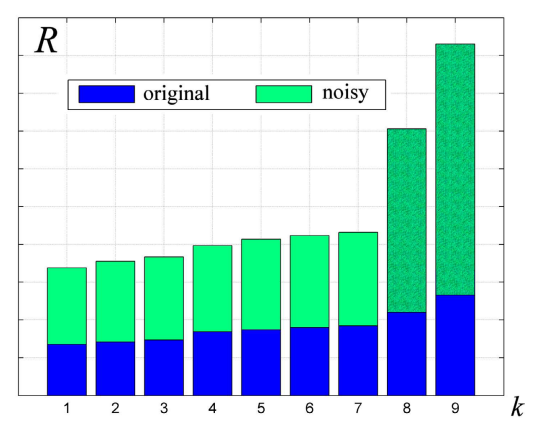

Fig. 2. Plot of the sorted aggregated distances $R$ of pixels from a $3 \times 3$ window of the original and noisy color test image. The filtering window contains two impulses (textured bars) which can be detected using the Fisher's linear discriminant.

$R_{i}^{\alpha}=\sum_{k=1}^{\alpha} \rho_{i}^{(k)}$ and the output of the generalized VMF is pixel, for which the trimmed sum of distances $R^{\alpha}$ is minimized.

The experiments performed on color test images indicate that the noise reduction properties of such a filtering design depend on the proper setting of the $\alpha$ parameter, as its optimal value is influenced by the kind of impulse noise and its intensity. Figure 1 exemplifies this observation showing the dependence of the PSNR image restoration measure on the value of $\alpha$ for a noisy LENA image contaminated with impulse noise.

In [9] the following criterion for determining the $\alpha$ parameter has been proposed

$$
\alpha=\max \alpha^{*} \quad \text { subject to }\left(\sum_{j=1}^{\alpha^{*}} \rho_{i}^{(j)}\right) \leq \rho_{i}^{(n)}, \boldsymbol{x}_{i} \in W,
$$

where $\rho_{i}^{(n)}$ is the largest distance between the central pixel $\boldsymbol{x}_{i}$ and its neighbors from $W$. This definition, although yields acceptable results is quite heuristic and therefore a new rule for the choice of $\alpha$ has been devised.

For the task of determining the value of $\alpha$, which can be treated as the cardinality of a cluster of pixels which were not disturbed by noise, we can make use of the Fisher's linear discriminant. Fisher's linear discriminant is a classification method that generally projects high-dimensional data onto a line and performs classification in this one-dimensional space. In our case, the dimensionality reduction of the color vectors, is performed by the calculation of the aggregated distances. Fig. 2 depicts the sorted aggregated distances of pixels in the filtering window of the original and noisy test image. Utilizing the Fisher's criterion the impulses injected by the noise process can be easily detected. 


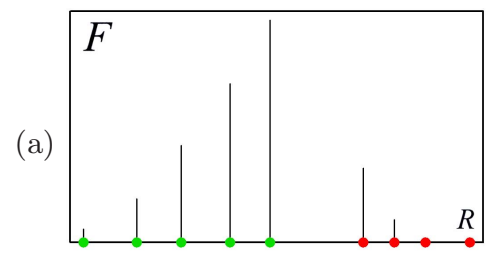

(b)

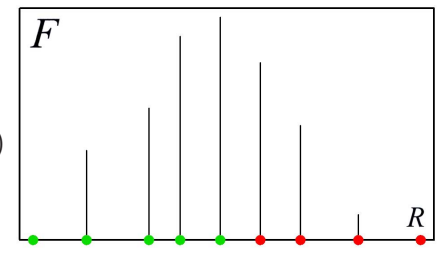

Fig. 3. Illustration of the Fisher's discrimination function for two distributions of the aggregated distances $R$. In each case, the maximum of the Fisher's discriminant determines the $\alpha$ value equal to 5 .

The goal of the discriminant is to maximize the distance between the means of two classes, while minimizing the variance within each class. This defines the Fisher criterion function $F_{(k)}$, where $k$ denotes the rank of the sorted sequence of the sorted $r_{(k)}$ values

$$
\begin{gathered}
F_{(k)}=\frac{\left[\mu_{1}(k)-\mu_{2}(k)\right]^{2}}{\sigma_{1}^{2}(k)+\sigma_{2}^{2}(k)}, \quad k=1, \ldots, n-1, \\
\mu_{1}(k)=\frac{1}{k} \sum_{i=1}^{k} R_{(i)}, \quad \mu_{2}(k)=\frac{1}{n-k} \sum_{i=k+1}^{n} R_{(i)},
\end{gathered}
$$

where the values $\mu_{1}$ and $\mu_{2}$ denote the mean values of the two classes of pixels and $\sigma_{1}^{2}$ and $\sigma_{2}^{2}$ stand for the variances of the aggregated distances in each of the two classes.

Figure 3 shows two plots of the Fisher linear discrimination function. As can be observed in Fig. 3 (a) the set of pixels in the filtering window is divided into two clusters. The $\alpha$ value is determined by the maximum of the Fisher's function. The division into two clusters is always performed, as shown in Fig. 3 (b), and as a result even in the absence of impulses a reasonable value of $\alpha$ is delivered by the Fisher's criterion, which guarantees the edge enhancing effect [9].

\section{$3 \quad$ Experiments}

For the evaluation of the efficiency of the proposed filter, a series of experiments has been performed utilizing natural and artificial color images contaminated by impulse noise. The applied noise model is defined as [6]

$$
\boldsymbol{x}_{i}=\left\{\begin{array}{cc}
\boldsymbol{o}_{i}, & \text { with probability } 1-p, \\
\left\{\rho_{i 1}, o_{i_{2}}, o_{i_{3}}\right\}, & \text { with probability } p_{1} p, \\
\left\{o_{i_{1}}, \rho_{i 2}, o_{i_{3}}\right\}, & \text { with probability } p_{2} p, \\
\left\{o_{i_{1}}, o_{i_{2}}, \rho_{i 3}\right\}, & \text { with probability } p_{3} p, \\
\left\{\rho_{i 4}, \rho_{i 4}, \rho_{i 4}\right\}, & \text { with probability } p_{4} p,
\end{array}\right.
$$

where $\boldsymbol{x}$ and $\boldsymbol{o}$ denotes the noisy and original color test image, $p$ is the sample corruption probability and $p_{1}, p_{2}, p_{3}$ are corruption probabilities of each color 


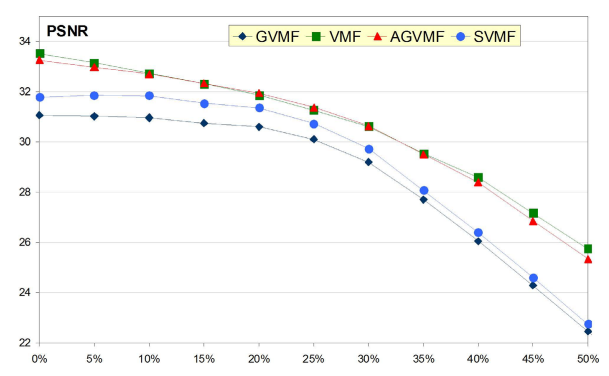

(a) PSNR

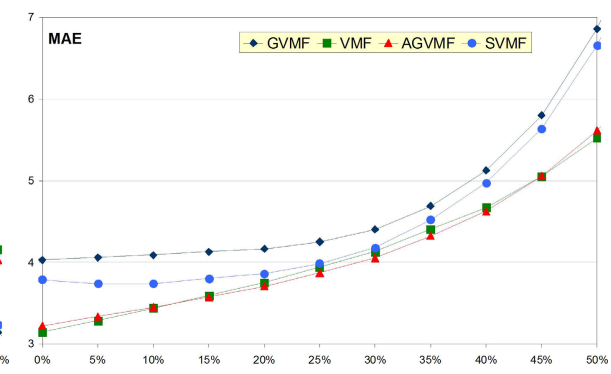

(b) MAE

Fig. 4. Dependence of the restoration quality measures on the noise intensity of the proposed AGVMF as compared with VMF, SVMF, 9] and GVMF with fixed parameter $\alpha=5$

channel, so that $\sum_{1}^{4} p_{k}=1$. The variables $\rho_{i k}, k=1, \ldots, 4$ take the value 0 or 255 with equal probability. In this work, the following parameter setting were used: $p_{k}=0.25$ for $k=1, \ldots, 4$.

For the measurement of the restoration quality, the Root Mean Squared Error (RMSE) expressed through the Peak Signal to Noise Ratio (PSNR) was used. For the evaluation of the detail preservation capabilities of the proposed filtering design the Mean Absolute Error (MAE) has been utilized.

The overall good noise reduction abilities of the proposed filtering design are presented in Fig. 4 which show the dependance of the PSNR and MAE on the noise intensity $p$ when restoring the LENA noisy image. As can be observed the efficiency of the proposed AGVMF is superior to that of the Sharpening VMF (SVMF) proposed in 9] and the Generalized VMF (GVMF) with fixed parameter $\alpha$. The noise reducing properties evaluated by means of PSNR and MAE are comparable with VMF, however the proposed AGVMF has the unique ability to sharpen the edges present in the color images. This feature is visible in Fig. 5 which depicts the filtering results delivered by the new filter as compared with the VMF, GVMF and SVMF.

The novel filtering technique can be applied for various tasks in which the noise reduction capabilities combined with the strong edge enhancing properties are beneficial. One of such applications is the analysis of the cDNA microarrays which quantify the genes expression levels [10]. As can be observed, the impulse noise is efficiently removed and the spots have sharp edges, which enable their reliable detection and estimation of the mean expression level calculated as an average intensity over the spot area.

The good efficiency of the proposed switching scheme can be observed in Fig. 6. which reveals the edge enhancing properties of the proposed noise reduction filter. The edge sharpening property can be utilized also for the enhancement of images which were not exposed to impulse noise. An example is provided by Fig. 7 which depicts parts of images of contact endoscopy. The improvement of the sharpness of edges can be evaluated when observing the output of the Vector 


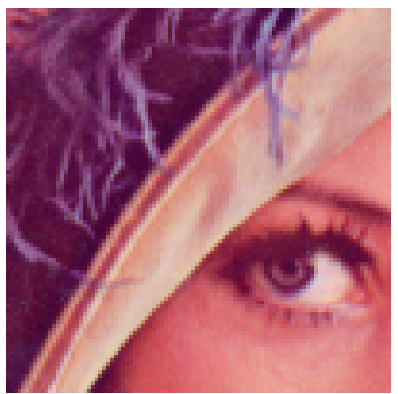

(a) LENA

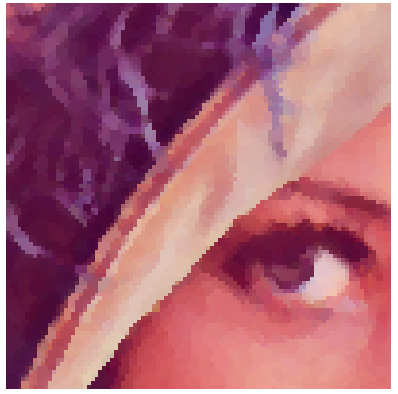

(d) AGVMF

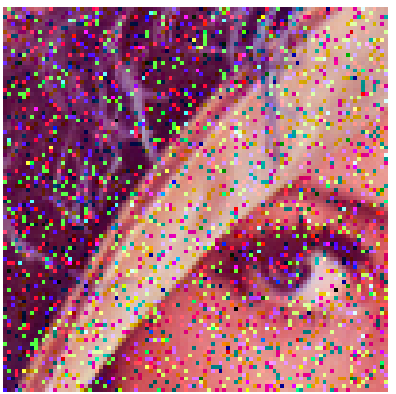

(b) NOISY

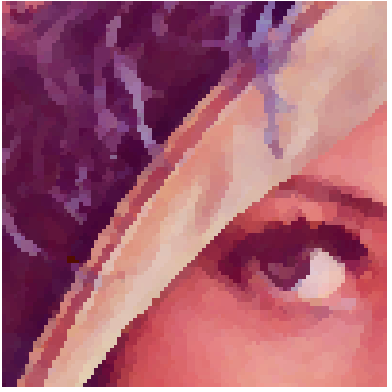

(e) $\mathrm{GVMG}_{\alpha=5}$

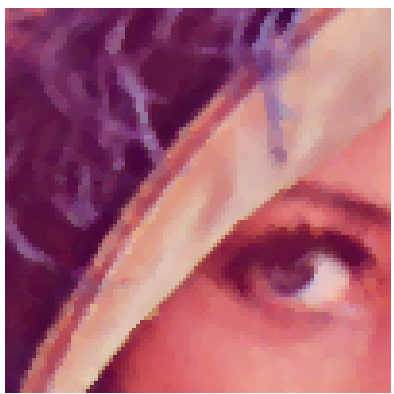

(c) $\mathrm{VMF}$

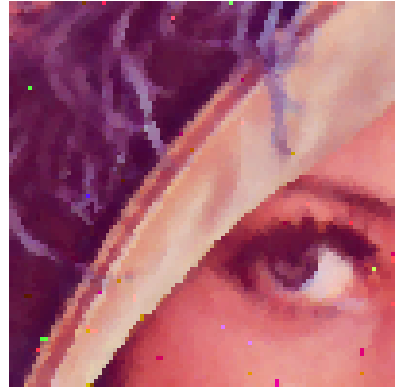

(f) SVMF

Fig. 5. Illustration of the efficiency of the proposed Adaptive Generalized VMF (AGVMF) as compared with the VMF, GVMF and SVMF

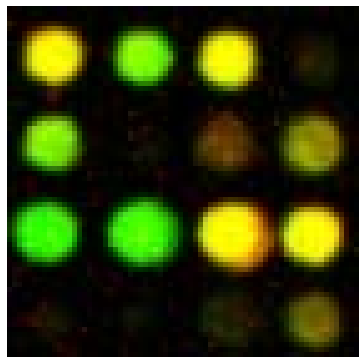

(a)

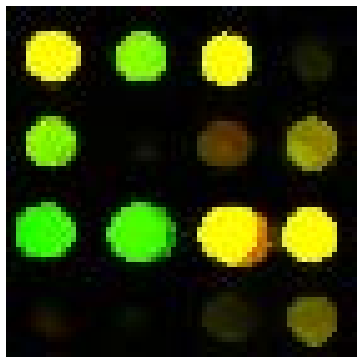

(b)

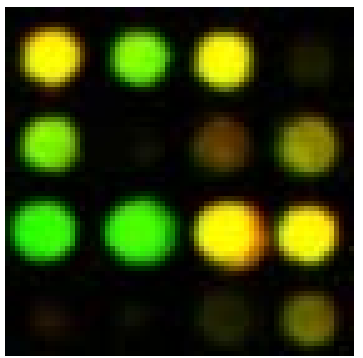

(c)

Fig. 6. Illustration of the noise reduction and edge enhancing capabilities of the new filter as compared with the VMF: (a) cDNA test image, (b) image restored with the proposed AGVMF, (c) VMF output.

Range (VR) edge detector defined as the distance between the vectors $\boldsymbol{x}_{(1)}$ and $\boldsymbol{x}_{(n)}$ from the filtering window [6]. As can be seen the quality of the edge maps is increased when the pre-filtering with the described PGVMF filter is performed. 


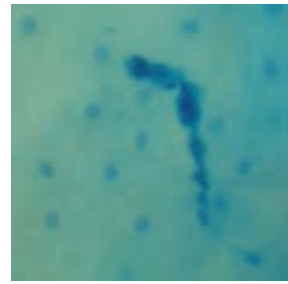

(a) Test Image

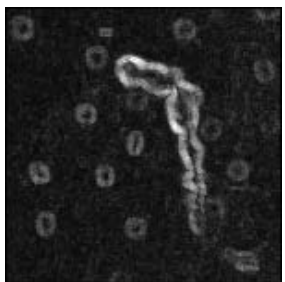

(e) VR

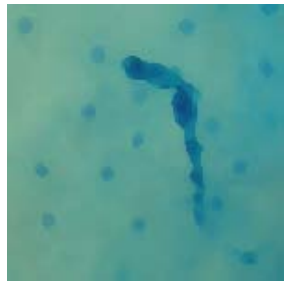

(b) AGVMF

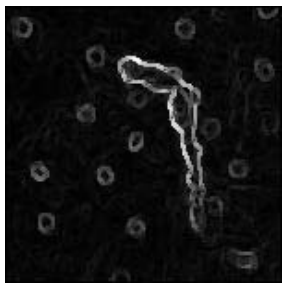

(f) VR

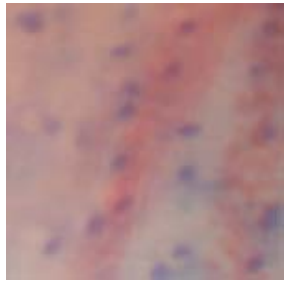

(c) Test Image

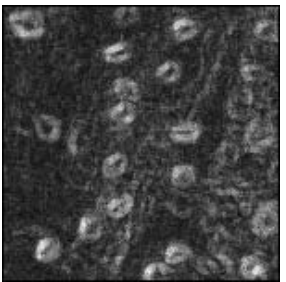

(g) VR

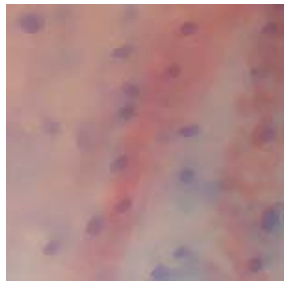

(d) AGVMF

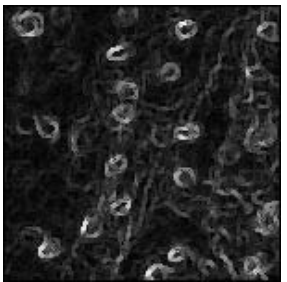

(h) VR

Fig. 7. Illustration of the edge enhancing property of the proposed filtering deign: (a) and (c) color images of contact endoscopy, (b) and (d) depict the new filter output. Below (e-h) the output of the vector range edge detector is presented.

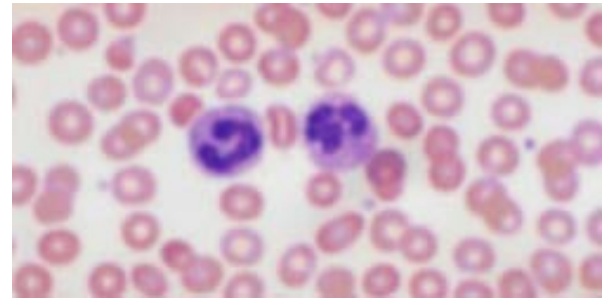

(a) original

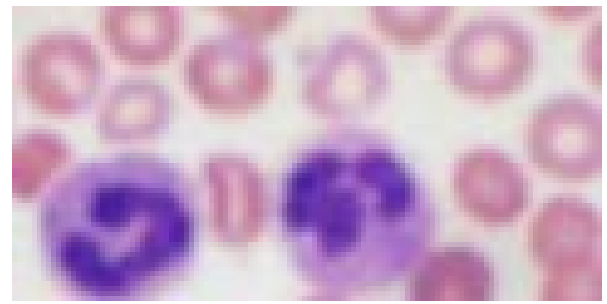

(c) original

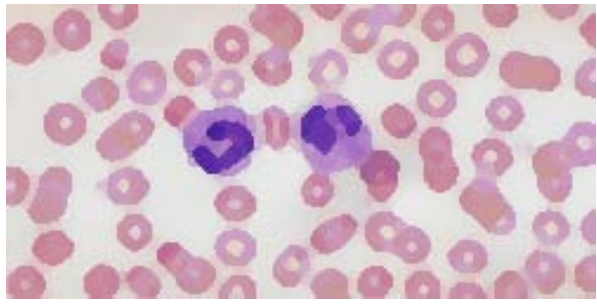

(b) enhanced

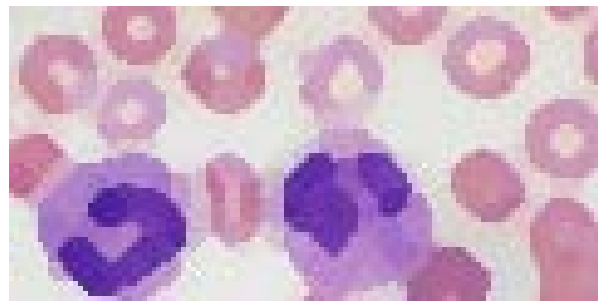

(d) enhanced

Fig. 8. Illustration of the edge enhancing properties of the proposed filtering design: (a) microscopic histology image, (b) output of the proposed adaptive filter after three iterations, (c-d) zoomed parts of the images above 
The final example, (Fig. 8) shows a microscopic blurry color image which can be enhanced using the proposed adaptive approach. This example indicates that the proposed algorithm can also be useful for the purpose of image segmentation.

\section{Conclusions}

In this paper, the properties of a novel adaptive filtering design has been examined. The proposed filter can be regarded as an adaptive generalization of the standard Vector Median Filter. Besides its excellent impulsive noise reducing capabilities, its unique feature is its ability to enhance color image edges by sharpenning their edges. This effect is really beneficial as in many applications sharp image edges are desired to enable the success of further image processing steps.

\section{References}

1. Astola, J., Haavisto, P., Neuvo, Y.: Vector Median Filters. Proc. of the IEEE 78(4), 678-689 (1990)

2. Perona, P., Malik, J.: Scale Space and Edge Detection Using Anisotropic Diffusion. IEEE Trans. on PAMI 12(7), 629-639 (1990)

3. Smolka, B., Plataniotis, K.N., Venetsanopoulos, A.N.: Nonlinear Techniques for Color Image Processing. In: Barner, K.E., Arce, G.R. (eds.) Nonlinear Signal and Image Processing: Theory, Methods, and Applications, pp. 445-505. CRC Press, Boca Raton (2004)

4. Schavemaker, J.G.M., Reinders, M.J.T., Gerbrands, J.J., Backer, E.: Image Sharpening by Morphological Filtering. Pattern Recognition 33(6), 997-1012 (2000)

5. Russo, F.: An Image Enhancement Technique Combining Sharpening and Noise Reduction. IEEE Trans. on Instrumentation and Measurement 51(4), 824-828 (2002)

6. Plataniotis, K.N., Venetsanopoulos, A.N.: Color Image Processing and Applications. Springer, Heidelberg (2000)

7. Lukac, R., Smolka, B., Martin, K., Plataniotis, K.N., Venetsanopoulos, A.N.: Vector Filtering for Color Imaging. IEEE Signal Processing Magazine 22(1), 74-86 (2005)

8. Kenney, C., Deng, Y., Manjunath, B.S., Hewer, G.: Peer Group Image Enhancement. IEEE Trans. on Image Processing 10(2), 326-334 (2001)

9. Lukac, R., Smolka, B., Plataniotis, K.N.: Sharpening Vector Median Filters. Signal Processing 87, 2085-2099 (2007)

10. Lukac, R., Plataniotis, K.N., Smolka, B., Venetsanopoulos, A.N.: A Multichannel Order-Statistic Technique for cDNA Microarray Image Processing. IEEE Trans. on Nanobioscience 3(4), 272-285 (2004) 